\title{
MSM in HIV-Prevention Trials are Sexual Partners With Each Other: An Ancillary Study to the EXPLORE Intervention
}

\author{
Matthew J. Mimiaga, ${ }^{1,2,8}$ Steven A. Safren, ${ }^{1,3}$ Dana Jones Benet, ${ }^{4,5}$ Marc W. Manseau, ${ }^{1,6}$ \\ Nancy DeSousa, ${ }^{1,7}$ and Kenneth H. Mayer ${ }^{1,7}$
}

Published online Dec. 6, 2005

The EXPLORE study evaluated a behavioral intervention to prevent HIV seroconversion among men who have sex with men (MSM). The present ancillary study enrolled 345 EXPLORE participants at one study site (Boston) and assessed high-risk sexual behavior with other EXPLORE participants. It also assessed sexual intentions across other EXPLORE participants, HIV-negative individuals, and unknown HIV serostatus partners. Thirty-one percent reported having sex with another EXPLORE participant: $27 \%$ unprotected receptive oral sex with ejaculation (UO), 30\% unprotected insertive anal sex (UIA), and $34 \%$ reported unprotected receptive anal sex (URA). Significant relationships between intentions to engage in UO, UIA, and URA, and type of partner emerged with intentions to engage in UO, UIA, and URA higher in HIV-negative partners, other EXPLORE participants, and unknownHIV serostatus partners. Future HIV-prevention studies recruiting MSM at increased sexual risk of HIV infection should address participants potentially becoming sexual partners with each other.

KEY WORDS: HIV-prevention trials; MSM; sexual behavior.

\section{INTRODUCTION}

Men who have sex with men (MSM) continue to represent one of the highest risk groups for HIV in the United States (Centers for Disease Control and Prevention; CDC, 2003). The EXPLORE study was

\footnotetext{
${ }^{1}$ Department of Research and Evaluation, Fenway Community Health, Boston, Massachusetts.

${ }^{2}$ Harvard University, School of Public Health, Boston, Massachusetts.

${ }^{3}$ Harvard Medical School/Massachusetts General Hospital, Boston, Massachusetts.

${ }^{4}$ Abt Associates Inc., Boston, Massachusetts.

${ }^{5}$ University of Michigan, School of Public Health, Ann Arbor, Michigan.

${ }^{6}$ Columbia University, College of Physicians and Surgeons, New York, New York.

${ }^{7}$ Brown University/Miriam Hospital, Providence, Rhode Island.

${ }^{8}$ Correspondence should be directed to Matthew J. Mimiaga, Research and Evaluation Department, Fenway Community Health, 7 Haviland Street, Boston, MA 02115; e-mail: mmimiaga@fenwayhealth.org.
}

the first randomized trial designed to test the efficacy of a behavioral intervention specifically for MSM in preventing the acquisition of HIV using HIV incidence as an outcome (Chesney et al., 2003; Koblin et al., 2003). While the intervention did not achieve a targeted level of efficacy (35\%) in preventing new HIV infections compared to semi-annual HIV voluntary counseling and testing, the study results suggest a possible modest benefit of the intervention in reducing new HIV infections (The EXPLORE Team, 2004). Further, the reporting of unprotected receptive anal sex with HIV-positive or unknown-status partners was significantly lower in the intervention group compared with the standard group (The EXPLORE Team, 2004).

The basis for conducting this ancillary study of sexual behaviors among and between EXPLORE participants arose from anecdotal observations by counselors at the Boston site that had enrolled 736 study participants for the main trial. Some study 
participants reported to their counselors in both the standard and intervention arms that they had engaged in risky sexual behaviors with other men in EXPLORE. They also described situations where they were less inclined to use condoms if their partner was in EXPLORE, and they perceived their risk of acquiring HIV to be less with these partners. Given the large number of MSM enrolled at the Boston site, we reasoned that it was likely that men in EXPLORE would be in situations where they could encounter each other and become sexual partners with each other. Moreover, participants knew that continued HIV seronegativity was required for continued participation in EXPLORE, and therefore determining one's EXPLORE status could be perceived as analogous to determining one's HIV status.

Recruitment methodologies may have also led to the increased likelihood of EXPLORE participants meeting each other. Recruitment strategies for HIV primary or secondary prevention trials are often centered on outreach at clubs, bars, sex clubs, health clubs, HIV/AIDS organizations and groups, and/or neighborhoods densely populated by gay men, where potential and current participants would likely have contact with each other (e.g., Reback et al., 2004; Koblin et al., 2003; Patterson et al., 2003). At the Boston site, $14 \%$ of EXPLORE participants were recruited through friends or word of mouth, however the most frequent recruitment venues were advertisements $(27 \%)$ and clubs and bars $(21 \%)$. Furthermore, it is not uncommon for trials of large magnitude to reward participation with incentives that bring study participants together. Overall the incentives for EXPLORE varied by city but in Boston included barbeque cookouts at parks, holiday themed dances, beach retreats, and dinner cruises. These incentives are another vehicle that allows for participant interaction. Given the intense publicity around the trial and targeted recruitment and retention efforts, we believe that most MSM in Boston were familiar with the EXPLORE study and knew it was for HIV-negative men.

The present study assessed the degree to which EXPLORE participants had engaged in high-risk sexual behavior with other EXPLORE participants. It also assessed whether intentions to engage in high-risk sexual behavior varied across other EXPLORE participants, HIV-negative individuals, and individuals of unknown HIV status. We hypothesized that a substantial percentage of EXPLORE participants would report having engaged in high-risk sexual activity with other EXPLORE participants, and that intentions to engage in unsafe sexual behav- iors would vary across types of partners (i.e., other EXPLORE participants, HIV-negative partners, and HIV-unknown status partners).

The Theory of Reasoned Action guided our logic behind assessing sexual intentions in addition to assessing sexual behaviors (Ajzen and Fishbein, 1980). Participants in this ancillary study were asked about intentions to have sex with other EXPLORE participants, and with partners who were not in EXPLORE with either HIV-negative or of unknownHIV status, in addition to assessing sexual behaviors. According to the Theory of Reasoned Action, intentions to engage in a behavior are a proximal predictor of a given behavior. The utility of the theory of reasoned action in predicting HIV prophylactic behavior (i.e., condom use intentions and behaviors) has been tested among a variety of populations, including MSM (Boldero et al., 1999; Fisher et al., 1995; McLaws et al., 1992; Ross and McLaws, 1992). In general, results of this research show that condom use is highly associated with intentions.

\section{METHODS}

\section{Participants and Procedures}

The present ancillary study began during the last 10 months of the EXPLORE study's duration. Four hundred and forty-one EXPLORE study participants were invited to participate during their pretest study visit and were eligible based on the following criteria: enrolled in the HIVNET 015 (EXPLORE), had a $24,30,36,42$, or 48 month pretest visit between September 2002 and July 2003, and were willing and able to provide informed consent. Three hundred and forty-five individuals consented and enrolled.

Inclusion criteria for the EXPLORE study were men who were 16 years or older, had anal sex with another man during the past year, and had not been involved in a mutually monogamous relationship in the past 2 years with a male partner who was HIV negative (Koblin et al., 2003). In the main EXPLORE trial, participants in both arms had HIV testing every 6 months. These assessments included two visitsone that involved HIV-pretest counseling, and one with HIV-posttest counseling. During the pretest visit, participants also completed a psychosocial assessment battery (Chesney et al., 2003) using the audio computer-assisted self-interview (ACASI) system. For the present study, because recruitment was staggered for the main trial, eligible participants were invited at the HIV-pretest counseling session for visit month $24,30,36,42$, or 48 of their participation. 


\section{Measures}

The present study reports on results of our ancillary questionnaire only. All enrolled EXPLORE ancillary study participants (i.e., standard and intervention participants) completed a survey about sexual behaviors with other EXPLORE participants, and intentions to engage in sexual behaviors with three types of male partners: (1) Partners who they knew were EXPLORE participants; (2) partners who they knew were HIV negative; and (3) partners whose HIV status was unknown. We adapted the ACASI sexual risk taking questions used in the EXPLORE main trial (Chesney et al., 2003) to tailor questions regarding sexual practices with other EXPLORE participants. These questions include the number of times the individual had engaged in receptive oral sex with ejaculation, insertive anal sex, and receptive anal sex with other EXPLORE participants, both with and without condoms. Regarding sexual intentions, participants rated the degree to which they agreed with three statements for each type of partner (i.e., other EXPLORE participants, HIV-negative partners, and HIV status unknown partners). These statements were: (1) You intend to have insertive anal sex with your partner and you don't use a condom; (2) you intend to have receptive anal sex with your partner and he doesn't use a condom; and (3) you intend to have receptive oral sex with your partner and he comes in your mouth. Participants were asked to rate their intentions on a four-point scale: 1 , very likely; 2 , somewhat likely; 3 , somewhat unlikely; and 4 , very unlikely.

\section{Data Analyses}

Descriptive statistics were calculated for demographic variables and sexual behaviors for all Boston EXPLORE participants. We used Chisquare global tests of independence for demographic variables (i.e., age, ethnicity, education, income, and employment) and the three outcome measures (i.e., unprotected receptive oral sex with ejaculation (UO), unprotected insertive anal sex (UIA), and unprotected receptive anal sex (URA)) to compare baseline differences between EXPLORE participants who were offered enrollment in this ancillary study and enrolled, those who were offered enrollment in the ancillary study and declined enrollment, and those who were enrolled in the EXPLORE main trial but were not eligible to enroll in this ancillary study.
For behavioral intentions, mean group comparisons were made using one-way analysis of variance (ANOVAs), followed by an examination of linear trends. Repeated-measures analyses of variance were conducted to compare each type of intentions across the three different types of partners.

\section{RESULTS}

Demographics of the ancillary study sample and Boston EXPLORE site are described in Table I. Out of 736 Boston EXPLORE study participants, $441(60 \%)$ were eligible and invited to participate in this study, of which 345 (78\%) enrolled. The remaining $96(22 \%)$ did not enroll due to lack of interest, time constraint and/or refusal. There were $295 \mathrm{EX}-$ PLORE participants who did not meet eligibility criteria for the ancillary study.

Baseline EXPLORE data show that participants who participated in the ancillary study were older, $\chi^{2}=37.71(\mathrm{df}=10), p<.001$; more likely to be White, $\chi^{2}=36.72(\mathrm{df}=10), p<.001$; more highly educated,

Table I. Baseline EXPLORE Ancillary Study and All Boston Site Demographic Data

\begin{tabular}{|c|c|c|c|c|}
\hline & $\begin{array}{c}\text { Ancillary } \\
\text { enrolled, } \\
N(\%)\end{array}$ & $\begin{array}{c}\text { Ancillary } \\
\text { declined, } \\
N(\%)\end{array}$ & $\begin{array}{c}\text { Ancillary } \\
\text { ineligi- } \\
\text { ble, } \\
N(\%)\end{array}$ & $\begin{array}{c}\text { All Boston } \\
\text { EXPLORE, } \\
N(\%)\end{array}$ \\
\hline \multicolumn{5}{|l|}{ Age (years) } \\
\hline $16-30$ & 101 (29) & $29(30)$ & $140(47)$ & $270(37)$ \\
\hline $31-40$ & $142(41)$ & $45(47)$ & $102(35)$ & 289 (39) \\
\hline $40+$ & $102(30)$ & $22(23)$ & $53(18)$ & $177(24)$ \\
\hline Mean $(S D)$ & $36.3(9.1)$ & $35.6(9.7)$ & $32.6(9.3)$ & $34.7(9.4)$ \\
\hline \multicolumn{5}{|l|}{ Race/ethnicity } \\
\hline White & $296(86)$ & $79(82)$ & $221(75)$ & $596(81)$ \\
\hline Black & $12(4)$ & $0(0)$ & $17(6)$ & $29(4)$ \\
\hline Hispanic & $18(5)$ & $11(12)$ & $49(17)$ & $78(11)$ \\
\hline Other & $19(6)$ & $6(6)$ & $8(2)$ & $33(5)$ \\
\hline \multicolumn{5}{|l|}{ Education } \\
\hline$\leq$ High school & $21(6)$ & $5(5)$ & 31 (11) & $57(8)$ \\
\hline Some college & $51(15)$ & $19(20)$ & $70(24)$ & 140 (19) \\
\hline $\begin{array}{l}\text { College } \\
\text { degree }\end{array}$ & $135(39)$ & $38(40)$ & $104(35)$ & $277(38)$ \\
\hline Post college & $138(40)$ & $34(35)$ & $89(30)$ & $261(36)$ \\
\hline \multicolumn{5}{|l|}{ Income $(\$)$} \\
\hline$<12,000$ & $29(8)$ & $12(13)$ & 57 (19) & $98(13)$ \\
\hline $12,000-29,999$ & $68(20)$ & $26(27)$ & $72(25)$ & $166(23)$ \\
\hline $30,000-59,999$ & $150(44)$ & $34(35)$ & 114 (39) & $298(41)$ \\
\hline $60,000+$ & $98(28)$ & $24(25)$ & $51(17)$ & $173(24)$ \\
\hline $\begin{array}{l}\text { Student } \\
\text { status-No }\end{array}$ & $295(86)$ & $79(82)$ & $224(76)$ & $598(81)$ \\
\hline $\begin{array}{l}\text { Recruitment by } \\
\text { friends }\end{array}$ & $48(14)$ & $5(5)$ & 47 (16) & $100(14)$ \\
\hline Total $N(\%)$ & $345(47)$ & $96(13)$ & $295(40)$ & 736 \\
\hline
\end{tabular}


$\chi^{2}=16.74(\mathrm{df}=6), p<.05$; had higher levels of income, $\chi^{2}=26.94(\mathrm{df}=6), p<.001$; and were more likely to be employed full-time, $\chi^{2}=29.50(\mathrm{df}=6)$, $p<.001$; and less likely to be students compared to those who refused participation or were not eligible, $\chi^{2}=9.65(\mathrm{df}=2), p<.01$. In terms of recruitment, ancillary study participants (14\%) and EXPLORE participants not eligible for the ancillary study $(16 \%)$ were more likely to have been recruited by friends than those declining participation in the ancillary study $(5 \%), \chi^{2}=7.15(\mathrm{df}=2), p<.05$.

There were no differences between the three groups (i.e., enrolled, declined, and not eligible) in the baseline UO, UIA, and URA measures for the HIV-negative and HIV-unknown status partners. Men who declined had lower rates of UO with HIVnegative partners than compared to those who enrolled or were not eligible, $\chi^{2}=5.58(\mathrm{df}=2), p=.06$. In addition, the Boston EXPLORE participants enrolled in the ancillary study were less likely to have reported UO, $\chi^{2}=9.27(\mathrm{df}=2), p<.01$, and URA, $\chi^{2}=8.91(\mathrm{df}=2), p<.05$, with HIV-positive partners at baseline than those who were eligible or refused participation. Due to missing data and one study withdrawal, 342 ancillary study surveys were analyzed.

\section{Self-Reported Sexual Behavior With Other EXPLORE Participants}

Ninety-six (31\%) of the 309 MSM who enrolled and answered all of the sexual behavior questions reported having at least one type of sex behavior with another EXPLORE participant during the study (309 of the 342 surveys analyzed had completed questions regarding sexual behavior with other EXPLORE participants). When asked about anal sex, with or without a condom, $67 \%$ of the 96 men reported engaging in insertive anal sex with another EXPLORE participant, and $74 \%$ reported engaging in receptive anal sex with another EXPLORE participant. Overall, these EXPLORE participants $(N=96)$ reported a mean of $2.8(S D=12.1)$ EXPLORE sexual partners since study enrollment.

Accordingly, of the 96 men, $27 \%(N=26)$ reported having UO with another EXPLORE participant at least once, $30 \%(N=29)$ reported UIA with another EXPLORE participant at least once, and $34 \%(N=33)$ reported URA with another EXPLORE participant at least once. See Table II for the mean number of instances of UO, UIA, and
Table II. Mean Scores of Unprotected Sexual Behavior with Another EXPLORE Participant

\begin{tabular}{llll}
\hline & $\begin{array}{l}\text { Unprotected } \\
\text { receptive oral } \\
\text { sex with } \\
\text { ejaculation } \\
(N=26)\end{array}$ & $\begin{array}{l}\text { Unprotected } \\
\text { insertive anal } \\
\text { sex }(N=29)\end{array}$ & $\begin{array}{l}\text { Unprotected } \\
\text { receptive anal } \\
\text { sex }(N=33)\end{array}$ \\
$\begin{array}{l}\text { Sexual } \\
\text { behavior }\end{array}$ & $5.1(21.8)$ & $9.5(45.7)$ & $8.0(35.1)$ \\
\hline $\begin{array}{l}\text { Other } \\
\text { EXPLORE } \\
\text { participants } \\
\text { mean }(S D)\end{array}$ & & & \\
\hline
\end{tabular}

URA with another EXPLORE participant since main trial enrollment. Because participants completed the questionnaire about sexual partners who were also EXPLORE participants at different times since enrollment in the main trial (i.e., either their $24,30,36,42$, or 48 month visit), we calculated an average score for each type of unprotected sexual behavior per 6-month period (i.e., we divided the total number of EXPLORE partners by the number of months in EXPLORE and multiplied that number by 6), as this was EXPLORE's frequency of pre- and post-HIV testing and counseling. The mean score for partners that were also in EXPLORE per 6-month period were: $1.0(S D=4.2), 1.9(S D=8.4)$, and $1.6(S D=7.5)$ for UO, UIA, and URA, respectively (these scores were calculated as an average for only those who had UO, UIA, or URA).

\section{Intentions to Engage in Sexual Behaviors Across Other EXPLORE, HIV-Negative, and HIV-Unknown Status Partners}

Intentions to engage in UO, UIA, and URA were compared regarding other EXPLORE participants, HIV-negative partners, and unknown-status partners $(N=342)$. Mean scores (and standard deviations) across all three types of partners are shown in Table III. Each of the three repeated-measure ANOVAs yielded significance: $F(2,335)=99.6$, $p<.001$ for intentions to engage in UO; $F(2$, $333)=86.4, p<.001$ for UIA; and $F(2,335)=76.7$, $p<.001$ for URA. For all three, a linear trend emerged, $F(1,336)=95.9,88.1,149.6$, respectively, all $p \mathrm{~s}<.001$; participants reported least intentions to engage in unsafe sex with partners of unknown status, they reported middle-level intentions (in between least likely intentions and most likely intentions) to engage in sex with other EXPLORE 
Table III. Mean Scores of Behavioral Intentions Across Types of Partners

\begin{tabular}{llll}
\hline & $\begin{array}{l}\text { Other } \\
\text { EXPLORE } \\
\text { participants } \\
\text { mehavioral }(S D)\end{array}$ & $\begin{array}{l}\text { HIV-negative } \\
\text { partners mean } \\
(S D)\end{array}$ & $\begin{array}{l}\text { Unknown- } \\
\text { status partners } \\
\text { mean }(S D)\end{array}$ \\
\hline $\begin{array}{l}\text { Unprotected } \\
\text { receptive } \\
\text { oral sex with } \\
\text { ejaculation }\end{array}$ & $3.29(0.96)$ & $2.84(1.10)$ & $3.53(0.80)$ \\
$\quad(N=337)$ & & & \\
$\begin{array}{l}\text { Unprotected } \\
\text { insertive } \\
\text { anal sex } \\
(N=335)\end{array}$ & $3.40(0.86)$ & $2.88(1.16)$ & $3.61(0.71)$ \\
$\begin{array}{l}\text { Unprotected } \\
\text { receptive } \\
\text { anal sex } \\
(N=337)\end{array}$ & $3.64(0.72)$ & $3.20(1.07)$ & $3.83(0.53)$ \\
\hline
\end{tabular}

Note. Behavioral intentions are on a scale from 1 to 4 with lower numbers indicating a higher likelihood.

${ }^{a} F(2,335)=99.6, p<.0001$.

${ }^{b} F(2,333)=86.4, p<.0001$

${ }^{c} F(2,335)=76.7, p<.0001$.

participants, and they rated most intentions to engage in unsafe sex with HIV-negative partners.

\section{DISCUSSION}

Almost one-third of the Boston EXPLORE participants enrolled in the ancillary study had sex with at least one other EXPLORE study participant. These results point to the importance of addressing "within-study sexual activity" (i.e., sexual activity with other study participants) in HIV-prevention trials, as these behaviors could affect both study outcomes and participants' health. In this study, intentions to engage in risky sex varied across partner type: intentions to engage in risky sex, such as URA, were highest for HIV-negative partners, in the middle range for partners who were also EXLORE participants, and then lowest for partners of unknown-HIV status. Supporting the "withinstudy sexual activity" hypothesis is the baseline data showing that only $14 \%$ of ancillary study participants were recruited by friends, and $31 \%$ of ancillary study participants reported at least one sexual experience with another EXPLORE participant. While some EXPLORE participants may have been familiar with one another before the study enrollment, the high number of reported sexual encounters after baseline and anecdotal evidence suggest that new relations were initiated.

These results can be viewed with respect to the Theory of Reasoned Action (Ajzen and Fishbein, 1980), in that sexual risk behavior with other EXPLORE participants may be influenced by greater intentions to have sex with other EXPLORE participants compared to intentions to have sex with HIVinfected or unknown-HIV status partners. In addition, these results are supported by theoretical components of the Health Belief Model (Rosenstock, 1974), which posits that engaging in behaviors necessary to avoid a disease depended on a person's perceived susceptibility to the disease, the perceived seriousness of the disease (perceived susceptibility and seriousness are often combined into perceived threat), and a weighing of the perceived benefits to taking action against perceived barriers to taking action. Because EXPLORE participation was contingent on remaining HIV-negative, and because the nature of the study involved regular testing and riskreduction counseling, EXPLORE participants may perceive their susceptibility to HIV and other sexually transmitted infections (STIs) as low when engaging in high-risk sexual behavior with other participants. Moreover, it could be that the extent to which "knowing" a partner's HIV status might possibly determine what kind of sex one actually has.

The possibility that EXPLORE participants viewed other EXPLORE participants as presenting a low risk for HIV infection, because a recent (every 6-months) HIV-seronegative test result was required to remain in the study, could have either benefits or barriers for HIV-prevention efforts. For some individuals, sex with only other EXPLORE participants could result in lower overall HIV and STI risk, yet for others, could result in higher risk. For example, it could result in lower risk if individuals were going to have unprotected sex anyway, but if they limited sex with other EXPLORE participants, it is likely that they would have lower risk than if they had sex with other MSM regardless of study participation or other indicators of ones partners' HIV status. This could be considered a calculated HIV risk-reduction strategy by study participants and by men in the community.

Admittedly, the information about HIV status being communicated is only as accurate as the last time the conveyor had unsafe sex, but they were HIV negative in the last 6 months. It is possible that in the mind of the person being told this, HIV risk with this person becomes significantly reduced and they 
are immediately a "less risky" partner than someone who either does not know their HIV status, or who does not wish to disclose their status; unfortunately, the data that was collected was not able to address this. Also, EXPLORE participants who engaged in sex with other EXPLORE participants might be significantly more likely to practice safer sex with each other, because both partners have been exposed to a prevention intervention, whereas the partners of unknown HIV status may have not been. However, the safety-risk ratio could also potentially be higher because individuals in EXPLORE, or any other large-scale HIV-prevention trial, can acquire HIV and other STIs during study involvement, especially if entry criteria require a history of high-risk sexual behavior. Inclusion criteria for the study involved at least some behavior that could put one at risk for HIV in the past 6 months. At baseline, 48.0 and $54.9 \%$ of individuals across sites reported unprotected receptive and insertive anal sex in the 6 months previous to enrollment, respectively (Koblin et al., 2003). Hence, these individuals, though HIV negative at enrollment, are considered those at risk for acquiring HIV and or other STIs. Moreover, it is important to mention that the EXPLORE main trial consent form made it clear that the trial was not proven to prevent HIV, and that participants must still avoid behaviors that would put them at risk for HIV infection. Prevention trial protocols that target individuals at risk for HIV may need to anticipate participants' assumptions about the safety of risky sex with other trial participants.

In light of the present study's findings, data from another behavioral risk-reduction trial also points to the importance of examining sexual behavior within trial participants in HIV-prevention studies (Imrie et al., 2001). This study to reduce sexual risk among MSM used a one-day group workshop as a cognitive behavioral intervention to reduce STIs. This intervention, like many others, brought participants together for the intervention itself; a same-time intervention may be less financially and temporally burdensome than individual treatment. Imrie et al. (2001) however, found that the individuals who received the group intervention actually had more new STIs post-study enrollment than those who did not. This study, however, did not report on whether men randomized to the intervention arm became sexually active with each other as a result of meeting during the group workshop. This issue could have confounded the interpretation of their results and other HIV-prevention studies.
In the present study, intentions to engage in high-risk sex with HIV-negative partners were higher than intentions to engage in high-risk sex with other EXPLORE participants. There are several possible explanations for this finding. Although having an HIV-negative serostatus was an inclusion criterion, other inclusion/exclusion criteria targeted individuals who were considered "risky" due to previous sexual behaviors. Therefore, partners categorized as "HIV negative" might be considered less risky than other EXPLORE participants. Also, it is unclear as to how individuals interpreted the phrase "HIV negative." To some, "HIV negative" could range from individuals who had been completely abstinent and had no risk whatsoever for HIV to individuals who had a recent negative HIV test, but may have engaged in high-risk behavior and were not outside their window period of the test.

Finally, the means (and standard deviations) for the intention to engage in high-risk sex measures suggest a skewed distribution and may point to additional phenomena. For example, some of the study participants disclosing their EXPLORE participation status may have "seen the light" and used their discussion of their study participation as a reinforcement of their intention to stay safe, compared to others who use it as a reinforcement of the fact that despite their previous risks, they are still uninfected. In the case of the latter group of individuals, pride or other factors may influence disclosure (i.e., I know how to manage my risks, even if I sometimes slip).

The results of the present study should be viewed with respect to the study's limitations. These limitations include a convenience sample, possible bias from participant self-report, and the fact that those who did seroconvert (and therefore may have been the most risky) during study participation did not participate in this ancillary study. Although financial incentives in the main EXPLORE trial (\$25 for pre- and post-HIV testing and counseling for visit months 6,12 , and 18 ; and $\$ 50$ for pre- and post-HIV testing and counseling for visit month 24 and each consecutive 6 month appointment thereafter) were used to encourage participant retention, we believe participant' reporting was not biased. This is because data collected for this study used an ACASI system, in which answers were not seen by study counselors (and participants were told this). This method has been shown to increase survey accuracy while simultaneously protecting the privacy of participants.

Further, as discussed above, participants in the present study were those not lost to follow-up, and 
therefore may not fully represent the target population. The results are also limited in that they represent a sample of individuals from one site of a multisite study, and may not be generalizable to other MSM across the country. Although men who were in a mutually monogamous relationship with another known HIV-negative MSM were excluded, it is possible that some of the sexual relationships with other EXPLORE partners existed prior to study involvement, especially because some men were recruited by snowball method (i.e., referral from friends), and this was not assessed. Because the cohort was recruited in a variety of ways that might have over sampled members of contiguous sexual networks (e.g., bars, readers of the same gay papers, snowball recruitment, etc.), it may not be surprising that many participants subsequently had sexual contact with other study members. This phenomenon may need to be considered in the design and analysis of future HIVprevention interventions, since it may lead to a statistical clustering effect, and could be a modifier in study outcomes. This cohort effect could have implications for possible skewing of results and will need to be considered before such studies are undertaken. Recruitment and analysis plans will need to anticipate or be designed to assess limit, or otherwise account for partner selection based on knowledge of study participation. Lastly, although it would be interesting to know how and where participants from the study who had sex with each other met—study sponsored events, or in bars, clubs, and backrooms (sex clubs) - this information was not obtained. A follow-up study could investigate this, as it might be instructive about the extent to which being part of the study encouraged actual verbal disclosure or indirect disclosure of HIV and study participation status. If, for example, being part of EXPLORE became equated in some networks with being "sexually safe" (i.e., HIV negative) for men beyond the reach of the study, then it could have important implications for how men use knowledge of their HIV status in negotiating condom use or other risk-reduction practices.

Most importantly, a substantial number of individuals in HIV-prevention studies may engage in HIV risk behavior with each other. The present study focused on MSM and sexual risk. These results point to the importance of, in future HIV-prevention trials, assessing and addressing within-study sexual activity in study design and interpretation of results.

\section{ACKNOWLEDGMENTS}

This work was supported by the HIV Network for Prevention Trials and sponsored by the US National Institute of Allergy and Infectious Diseases and the National Institute on Alcohol Abuse and Alcoholism, of the National Institutes of Health, Department of Health and Human Services, through contract N01 AI35176 with Abt Associates Inc.; contract N01 AI45200 with the Fred Hutchinson Cancer Research Center. In addition, this work was supported by the HIV Prevention Trials Network and sponsored by the National Institute of Allergy and Infectious Diseases, the National Institute of Child Health and Human Development, the National Institute on Drug Abuse, the National Institute of Mental Health, and the Office of AIDS Research, of the National Institutes of Health, US Department of Health and Human Services, through a cooperative agreement with Family Health International (cooperative agreement 5 U01 AI46749) with a subsequent subcontract to Abt Associates Inc. and a cooperative agreement U01 AI48040 to the Fenway Community Health Center. Special thanks to Marla Husnik for reviewing the manuscript.

\section{REFERENCES}

Ajzen, I., and Fishbein, M. (1980). Understanding attitudes and predicting social behavior. Englewood Cliffs, NJ: Prentice Hall.

Boldero, J., Sanitioso, R., and Brain, B. (1999). Gay Asian Australians' safer-sex behavior and behavioral skills: The predictive utility of the theory of planned behavior and cultural factors. Journal of Applied Social Psychology, 29, 2143-2163.

Centers for Disease Control and Prevention (2003). HIV/AIDS surveillance report: Cases of HIV infection/AIDS in the United States, 2002, Vol. 14.

Chesney, M. A., Koblin, B. A., Barresi, P. J., Husnik, M. J., Celum, C. L., Colfax, G., Mayer, K. H., McKirnan, D., Judson, F. N., Huang, Y., Coates, T. J., and the EXPLORE Study Team. (2003). An individually tailored intervention for HIV prevention: Baseline data from the EXPLORE study. American Journal of Public Health, 93, 933-938.

Fisher, W. A., Fisher, J. D., and Rye, B. J. (1995). Understanding and promoting AIDS-preventive behavior: Attitude-behavior correspondence. Health Psychology, 14, 255-264.

Imrie, J., Stephenson, J. M., Cowan, F. M., Wanigaratne, S., Billington, A. J. P., Copas, A. J., French, L., French, P. D., and Johnson, A. M. (2001). A cognitive behavioural intervention to reduce sexually transmitted infections among gay men: Randomized trial. British Medical Journal, 322, 14511456.

Koblin, B. A., Chesney, M. A., Husnik, M. J., Bozeman, S., Celum, C. L., Buchbinder, S., Mayer, K. H., McKirnan, D., Judson, F. N., Huang, Y., Coates, T. J., and the EXPLORE Study Team. (2003). High-risk behaviors among men who have sex with 
men in 6 US cities: Baseline data from the EXPLORE study. American Journal of Public Health, 93, 926-932.

McLaws, M. L., Oldenburg, B., and Ross, M. W. (1992). Application of the theory of reasoned action to the measurement of condom use among gay men. Sydney, Australia: Albion AIDS Centre, Division of Medicine, Prince of Wales Hospital.

Patterson, T. L., Shaw, W. S., and Semple, S. J. (2003). Reducing the sexual risk behaviors of $\mathrm{HIV}^{+}$individuals: Outcome of a randomized controlled trial. Annals of Behavioral Medicine, $25,137-145$

Reback, C. J., Larkins, S., and Shoptaw, S. (2004). Changes in the meaning of sexual risk behavior among gay and bisexual male methamphetamine abusers before and after drug treatment. AIDS and Behavior, 8, 87-98.

Rosenstock, I. (1974). Historical origins of the health belief model. Health Education Monographs, 2(4).

Ross, M. W., and McLaws, M. L. (1992). Subjective norms about condoms are better predictors of use and intentions than attitudes. Health Education Research, 7, 335-339.

The EXPLORE Study Team. (2004). Effects of a behavioural intervention to reduce acquisition of HIV infection among men who have sex with men: The EXPLORE randomized controlled study. The Lancet, 364, 41-50. 